\title{
Effect of Anti-Inflammatory Eye Drops on Bacterial Keratitis
}

\author{
Hwa-Rang Lim and Jae Woong Koh* \\ Department of Ophthalmology, Gwangju, Republic of Korea \\ *Corresponding author: Jae Woong Koh, Department of Ophthalmology, 365 Pilmun-daero, Dong-gu, Gwangju, \\ Republic of Korea
}

ARTICLE INFO

Received: 慧 June 12, 2019

Published: 彗 June 21, 2019

Citation: Hwa-Rang Lim, Jae Woong Koh. Effect of Anti-Inflammatory Eye Drops on Bacterial Keratitis. Biomed J Sci \& Tech Res 19(1)-2019. BJSTR. MS.ID.003240.

Keywords: Bacterial keratitis; NSAID; Steroid; Anti-inflammatory treatment

Abbreviations: OD: Oculus Dexter; OS: Oculus Sinister; BCVA: Best Corrected Visual Acuity; log MAR: Logarithm of the Minimum Angle of Resolution; NSAID: Non-Steroidal Anti-Inflammatory Drug; DALK: Deep Anterior Lamellar Keratoplasty; PAF: Platelet-Activating Factor
ABSTRACT

Aim

To evaluate and compare the effect of non-steroidal anti-inflammatory drug (NSAID) eye drop and steroid eye drop on bacterial keratitis patient's response to antibiotics.

\section{Methods}

From Sep. 2017 to Sep. 2018, 60 patients diagnosed with bacterial keratitis were enrolled. 20 patients who did not want anti-inflammatory eye drop were treated with antibiotics. 20 patients were treated with steroid eye drop and 20 patients were treated with NSAID eye drop. Patients were evaluated for the ulcer size, ulcer location, visual acuity, recurrence rate. Anti-inflammatory treatment was considered when corneal epithelial defect, corneal infiltration and anterior chamber inflammation were decreased after antibiotic eye drops use. In case of re-aggravation, anti-inflammatory treatment was discontinued.

\section{Results}

There was no significant difference in mean age, sex, ulcer size, ulcer location, and initial visual acuity between three groups. All 60 patients diagnosed unilateral bacterial keratitis. After 3-month, anti-inflammatory treatment groups showed significant improvement of best corrected visual acuity (BCVA) compared to the group treated with antibiotics $(p=0.045)$. However, there was no statistically significant difference in the 3 -month BCVA between NSAID group and steroid group ( $\mathrm{P}=0.743)$. In NSAID treated group, one of 20 eyes (5.0\%) showed re-aggravating inflammation, while steroid treated group, 3 of 20 eyes (15.0\%) showed re-aggravating inflammation. Recurrence rate was lower in NSAID treated group, however, there was no statistically significant difference between two groups $(\mathrm{P}=0.605)$.

\section{Conclusion}

In bacterial keratitis, NSAID has an inflammatory control effect like steroid and extra-advantage on lower recurrence rate.

\section{Introduction}

Bacterial keratitis is a severe infectious disease of cornea. Despite adequate antibiotic treatment, it is a serious disease that may cause a permanent visual loss due to corneal opacity, corneal neovascularization and corneal perforation ultimately leading to permanent visual impairment $[1,2]$. Cornea epithelial defect and infiltration of corneal stroma cause a range of symptoms including a visual loss, ocular pain, hyperemia, tearing, and glaring. The causes of corneal ulcers are ocular trauma, contact lens use, ocular surface disease, eyelid and eye surgery, HIV, immunodeficiency disease, and use of systemic steroids [3]. In a hospital study conducted in 2001, the incidence of bacterial keratitis in Korea has increased compared to the past. In addition, the proportion of women has increased and has changed to younger ages. This is due to the increased use of contact lenses in young women [4]. In bacterial keratitis, it is 
important to identify bacteria by microbial staining and culture to confirm sensitivity of antibiotics. Empirical broad-spectrum antibiotics should be used until obtaining results $[5,6]$. However, if the inflammation is not controlled despite the early treatment, the ulcer may lead to corneal opacity and vision loss. After treatment, best corrected visual acuity (BCVA) depends on the degree of inflammatory reaction and size and location of corneal ulcer [7].

In addition, if the visual acuity decreases due to corneal opacity and neovascularization after complete cure of the corneal ulcer, surgical treatment such as corneal transplantation or deep anterior lamellar keratoplasty (DALK) will be required. Anti-inflammatory treatment in bacterial keratitis is one of the useful methods to decrease corneal opacity, though it is still controversial, since it can delay the wound recovery and increase bacterial activity by inhibiting the local immune response [8-12] Aronson S et al. reported the use of high-dose topical steroids in 16 patients with bacterial corneal ulcers. Seven out of 16 had the final visual acuity of 20/60 or better Carmichael T et al. [13] carried out 18 months retrospective study which 40 bacterial corneal ulcer patients. Two groups were compared: one was treated with antibiotic only and the other with antibiotic plus steroid. There was no statistical difference in visual outcome between the two groups. However, the group which used the steroid, had better average cure rate and final visual acuity. In addition, there was no difference in the incidence of complications between the two groups [14]. Anti-inflammatory treatment is a relatively simple and economical treatment method that can reduce the corneal opacity and improve the final visual acuity. However, no comparison between the therapeutic effects of nonsteroidal anti-inflammatory drug (NSAID) eye drops and steroid eye drops has been reported. Therefore, we compare the recurrence rate and therapeutic effect of NSAID treatment and steroid treatment in bacterial corneal ulcer patients.

\section{Materials and Methods}

Between September 2017 and September 2018, 60 patients clinically diagnosed with bacterial keratitis at 00 university college of medicine were enrolled. This study was approved by the Institutional Review Board (IRB) of the 00 University college of medicine. Patients who did not want to be treated with antiinflammatory drops were treated with antibiotics. Randomized, double-masked study was conducted in patients who were treated with NSAID and steroid eye drop. Major exclusion criteria included corneal perforation or impending perforation, evidence of fungus on potassium hydroxide preparation, evidence of acanthamoeba by stain, evidence of herpetic keratitis by history or examination, use of a topical corticosteroid or systemic prednisolone during the present ulcer, previous penetrating keratoplasty. Corneal ulcer location was divided into three zones; it was considered 'central' if the ulcer was placed within $3 \mathrm{~mm}$ from the center of the cornea, 'peripheral' if the ulcer was placed over $3 \mathrm{~mm}$ from the center of the cornea and 'paracentral' if the ulcer was placed in the border. The size of the ulcer was obtained by multiplying X-axis of Y-axis that is perpendicular to X-axis. Clinically, when bacterial keratitis was suspected, corneal debridement was performed prior to antibiotic treatment. And microorganism staining and culture were performed to identify causative organisms and the sensitivity of antibiotics.

For all patients, Fortified Cefazolin 5\% and Fortified Tobramycin $1.4 \%$ were used 8 times a day, and Atropine Sulfate 1\% (IsoptoAtropine ${ }^{\circledR}$, Alcon, Fort Worth, TX, USA) were used 3 times a day, and Hyaluronic acid $0.3 \%$ (Hyaluni®, Daejeon, Seoul, Korea) was used 8 times a day at first visit. Empirical antibiotic treatment was performed for at least 5 days before laboratory confirmation. BCVA, corneal epithelial defect, corneal infiltration and anterior chamber inflammation were re-evaluated every day while using empirical antibiotics. Additional anti-inflammatory treatment was carried out in patients who well responded to the antibiotic treatment and want to use. Bromfenac sodium hydrate 0.1\% (Bronuck $®$, Daejeon, Seoul, Korea) applied 2 times per day for 4 weeks with antibiotics in NSAID group. Fluorometholone $0.1 \%$ (Flumetholon $®$, Santen, Osaka, Japan) applied 4 times per day for 1 weeks with antibiotics in Steroid group, then 3 times a day for 1 week, and then twice a day for 1 week, and then once a day for 1 week. BCVA, corneal epithelial defect, corneal infiltration and anterior chamber inflammation were evaluated after the anti-inflammatory treatment to observe the adverse effect. When adverse effects such as aggravation of inflammation, increased epithelial defect, and decreased visual acuity were observed, it was considered as a recurrence and anti-inflammatory treatment was discontinued. Statistical analysis was carried out by chi-square test, Fisher exact test, ANOVA test. Two-way mixed ANOVA was used to compare the improvement of visual acuity. Statistical analysis was conducted using SPSS statistical program (version 18.0, SPSS Inc., Chicago, IL, USA) and $\mathrm{P}$ values $<0.05$ were considered statistically significant.

\section{Results}

60 patients were enrolled. 20 patients were treated with only antibiotics and 40 treated with anti-inflammatory drop. Twenty of the 40 patients were randomized receive NSAID eye drop, and 20 received steroid eye drop. Overall, enrollment characteristics were well balanced between three groups; sex, mean age, ulcer size, location, initial BCVA (Table 1). After 3-month, anti-inflammatory treatment groups showed significant improvement of BCVA compared to the group treated with antibiotics $(p=0.045)$. Since there was no statistically significant difference between NSAID group and steroid group (Figure 1) ( $\mathrm{p}=0.889)$. 3-month BCVA was $\log$ MAR $0.25 \pm 0.21$ in antibiotics group, and log MAR $0.14 \pm 0.17$ in NSAID group, and log MAR $0.18 \pm 0.32$ in steroid group. NSAID treatment group showed lower recurrence rates than steroid group. However, there was no statistically significant difference between the two groups ( $\mathrm{p}=0.332$ ) (Figure 2). One eye (5.0\%) of 20 eyes in NSAID group and 3 eyes (15.0\%) of 20 eyes in steroid group showed re-aggravation of inflammation (Figures $3 \& 4$ ). 


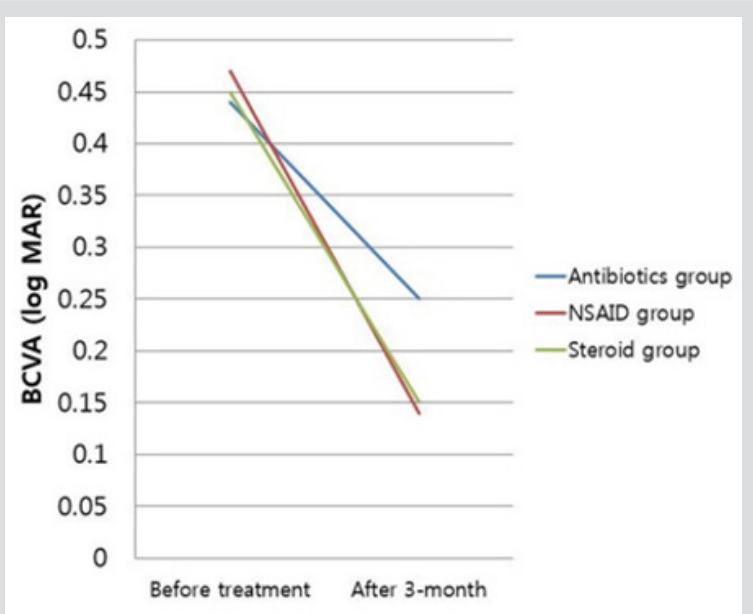

*Two-way mixed ANOVA

BCVA, best corrected visual acuity; NSAID, non-steroidal anti-inflammatory drug

Figure 1: The BCVA at baseline and at 3 months after treatment. There was statistically significant difference in the improvement of visual acuity in both groups $\left(\mathrm{p}=0.045^{\star}\right)$ but there was no statistically significant difference in visual acuity improvement between the two groups $\left(\mathrm{p}=0.889^{*}\right)$.

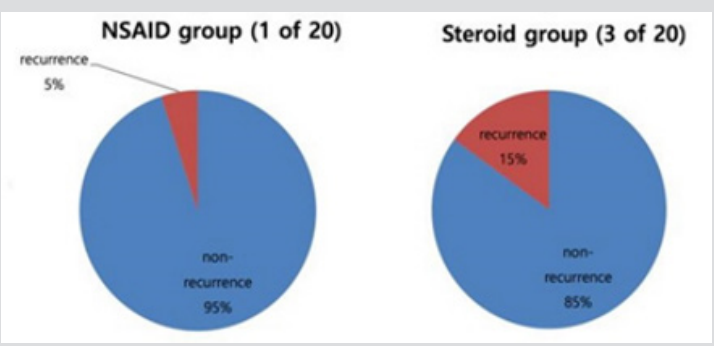

*Fisher exact test.

Figure 2: Comparison of the recurrences between the NSAID eye drops treatment group and Steroid treatment group. NSAID treatment group showed lower recurrence rate but there was no statistically significant difference between the two groups $\left(\mathrm{p}=0.332^{*}\right)$.

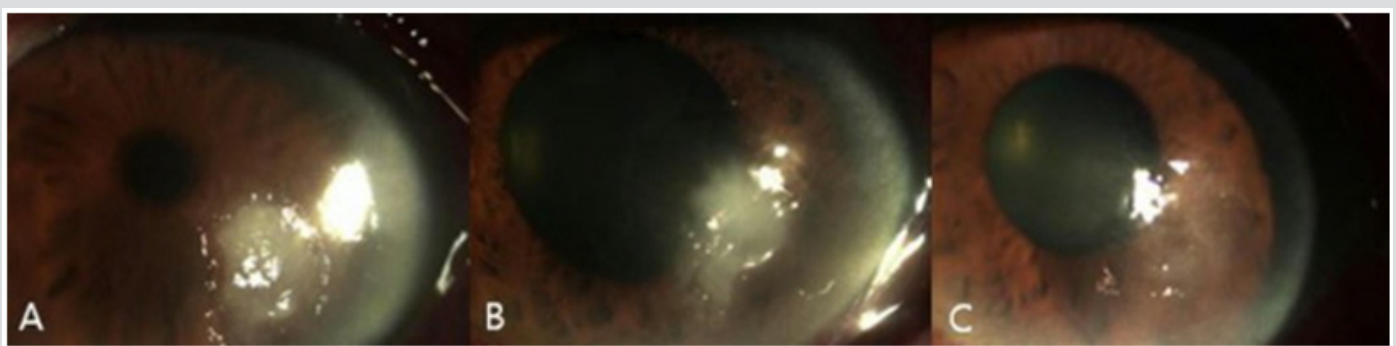

Figure 3: Anterior segment photograph of 42-years-old in NSAID group. (A) At presentation, corneal epithelial defect and infiltration at 5 o'clock. (B) After 4days treatment with antibiotics, epithelial defect almost healed and infiltration decreased. (C) 1monthafter anti-inflammatory treatment, 5 o'clock cornea lesion completely healed.

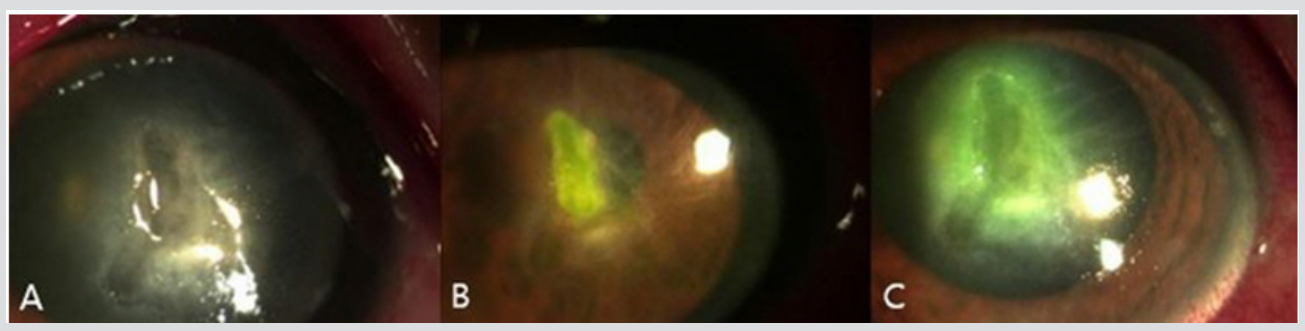

Figure 4: Anterior segment photograph of 63-years-old in steroid group. (A) At presentation, corneal epithelial defect and infiltration at center of cornea. (B) After 7days treatment with antibiotics, corneal infiltration and epithelial defects were decreased. (C) 2 days after anti-inflammatory treatment, size of the epithelium defect was increased and the corneal infiltration re-aggravation and anti-inflammatory treatment was stopped. 
Table 1: Demographic data of patients about sex, age, study eye, ulcer area, ulcer location, visual acuity of previous treatment.

\begin{tabular}{|c|c|c|c|c|}
\hline & Antibiotics group & NSAID group & Steroid group & P value \\
\hline Sex (Male: Female) & $9: 11$ & $12: 8$ & $10: 10$ & $0.810^{*}$ \\
\hline Age & $49.37 \pm 15.62$ & $46.57 \pm 20.61$ & $45.78 \pm 22.13$ & $0.653 \#$ \\
\hline Study eye (OD: OS) & $11: 9$ & $9: 11$ & $7: 13$ & $0.885^{*}$ \\
\hline Ulcer area (mm ${ }^{2}$ ) & $2.96 \pm 1.81$ & $3.06 \pm 1.51$ & $3.16 \pm 1.49$ & $0.691 \#$ \\
\hline $\begin{array}{c}\text { Ulcer location (central: } \\
\text { paracentral: peripheral) }\end{array}$ & $10: 3: 7$ & $9: 6: 5$ & $10: 6: 4$ & $0.510^{*}$ \\
\hline BCVA (log MAR) & $0.44 \pm 0.22$ & $0.47 \pm 0.25$ & $0.45 \pm 0.42$ & $0.908 \#$ \\
\hline
\end{tabular}

*Chi-square test, \# ANOVA test

\section{Discussion}

Even with early and aggressive treatment, Bacterial keratitis may cause permanent visual loss by leaving corneal opacity. In addition to direct corneal damage by bacteria, the immune reaction to inflammation also weakens the normal structure of the cornea. $\mathrm{T}$ cells and macrophages react to bacteria to produce cytokines such as IL-1, IL-2 and Tumor necrosis factor, promoting neutrophil migration and degranulation [15]. In particular, the plateletactivating factor (PAF) can elevate metalloproteinase and cause more necrosis [16]. Immune reaction to these bacteria ultimately contributes to decreased corneal thickness and increased corneal opacity. Therefore, anti-inflammatory treatment has been used not only for bacterial corneal ulcers but also for herpes keratitis, corneal opacity and scarring after refractive surgery [17]. In addition, anti-inflammatory treatment can reduce angiogenesis of the cornea by reducing inflammatory factors such as prostaglandin, which cause vasoconstriction [16]. Anti-inflammatory treatment for bacterial corneal ulcers has been tried and discussed by many studies. Wilhelmus K. carried out meta-analysis for various studies from 1950 to 2000, but failed to conclude on the efficacy of topical steroid use in bacterial corneal ulcers [18] Stern G suggested that anti-inflammatory treatment for bacterial corneal ulcers should be combined with antibiotic treatment and that anti-inflammatory treatment should be initiated at a time when pathogens are identified or at least for a few days reacting to initial antibiotic treatment Srinivasan M et al. [19] conducted a clinical trial with 500 patients. Patients diagnosed with corneal ulcers used moxifloxacin $0.5 \%$ for 48 hours. Subsequently, anti-inflammatory group used prednisolone sodium phosphate $1 \%$ while the control group used placebo. After 3 months, there was no difference in BCVA, corneal wound size and corneal perforation ratio between the anti-inflammatory group and placebo groups.

However, the efficacy of anti-inflammatory treatment in patients with low initial visual acuity or ulceration at the center of the cornea was reported. In patients with visual acuity less than finger count, BCVA in the anti-inflammatory group was 1.7 line higher than placebo group at 3 months after treatment. In the group with corneal ulcers located within $4 \mathrm{~mm}$ of the corneal center, BCVA in the anti-inflammatory group was 2 lines higher than placebo group at 3months after treatment [20]. Additional studies were reported for time of application of anti-inflammatory therapy. Patients who started anti-inflammatory treatment 2-3 days after antibiotic treatment, the anti-inflammatory group showed an average improvement of 1.7 lines better than the placebo group. However, there was no difference in visual acuity between the two groups in patients who started anti-inflammatory treatment after 3 days of antibiotic treatment [21]. In conclusion, anti-inflammatory treatment in bacterial keratitis is safer to initiate after 48 hours of adequate antibiotic treatment for patients with low visual acuity or corneal ulcer placed in the center of cornea. On the other hand, side effect of anti-inflammatory treatment may increase the bacterial activity and decrease the corneal thickness by melting the corneal parenchyma, which increases the risk of corneal perforation [22]. Animal studies have shown that when corneal injury is present, local steroid administration can reduce wound strength and delay corneal epithelial regeneration [23,24].

In addition, various side effects such as increased intraocular pressure, posterior capsular cataract, tear film instability and crystalline keratopathy have been reported [25]. Because of the possibility of these various side effects, the authors observed daily BCVA, intraocular pressure and slit lamp examinations for 3 days after the start of anti-inflammatory treatment. Thereafter, examinations were performed every week until the end of antiinflammatory treatment. In this study, there were no patients with corneal perforation and elevated intraocular pressure. But aggravation of inflammation was observed in one $(5.0 \%)$ of 20 eyes with NSAID treatment group, and in $3(15.0 \%)$ of 20 eyes with steroid treatment group. The mean incidence of complications higher than $6 \%$ reported by Srinivasan $\mathrm{M}$ et al. The high recurrence rate in our study is due to the small number of patients. The antiinflammatory treatment in corneal ulcer has several advantages. First, it is more economical to treat corneal opacity than the surgical method. Second, it is easy to apply and the patient compliance is high. Third, complications and recurrence rates are low. Fourth, improvement of final visual acuity after treatment can reduce the necessity of surgical treatment such as penetrating keratoplasty. Serious complications such as perforation, elevated intraocular pressure and worsening of inflammation are possible, but they can be prevented and treated through close observation after the initiation of anti-inflammatory treatment. 


\section{Conclusion}

In this study, the authors compared the treatment effects of two anti-inflammatory eye drops, but there was no statistically significant difference between the two groups in the recurrence rate and degree of visual improvement. However, significant improvement in visual acuity was observed in both antiinflammatory treatment groups, and especially a lower recurrence rate was observed in NSAID treatment group. In conclusion, nonsteroidal anti-inflammatory eye drops in bacterial corneal ulcer is an effective treatment with equivalent effect on recurrence rate and visual improvement compared with steroid eye drops.

\section{References}

1. Whitcher JP, Srinivasan M, Upadhyay MP (2001) Corneal blindness: a global perspective. Bull World Health Organ 79(3):214-221.

2. Pepose JS, Wilhelmus KR (1992) Divergent approaches to the management of corneal ulcers. Am J Ophthalmol 114(5): 630-632.

3. Ibrahim YW, Boase DL, Cree IA (2009) Epidemiological characteristics, predisposing factors and microbiological profiles of infectious corneal ulcers: the Portsmouth corneal ulcer study. British Journal of Ophthalmology 93(10):1319-1324.

4. Yun YS, Tchah SW, Joo CK (2001) Epidemiology of Infectious Keratitis (2): A multi-center study. J Korean Ophthalmol Soc 42: 228-247.

5. Dahlgren MA, Lingappan A, Wilhelmus KR (2007) The clinical diagnosis of microbial keratitis. American Journal of Ophthalmology 143(6): 940944.

6. McLeod SD, LaBree LD, Tayyanipour R, Flowers CW, Lee PP, et al. (1995) The Importance of Initial Management in the Treatment of Severe Infectious Corneal Ulcers. Ophthalmology 102(12): 1943-1948.

7. Henry CR, Flynn HW, Miller D, Richard KF, EC Alfonso (2012) Infectious keratitis progressing to endophthalmitis: a 15-year study of microbiology, associated factors, and clinical outcomes. Ophthalmology 119(12): 2443-2449.

8. Chung JH, Kang YG, Kim HJ (1998) Effect of 0.1\% dexa-methasone on epithelial healing in experimental corneal alkali wounds: morphological changes during the repair process. Graefe's Arch Clin Exp Ophthalmol 236(7): 537-545.

9. Tomas-Barberan S, Fagerholm P (1999) Influence of topical treatment on epithelial wound healing and pain in the early postoperative period following photorefractive keratectomy. Acta Ophthalmol Scand 77(2): 135-138.

\section{ISSN: 2574-1241}

DOI: 10.26717/BJSTR.2019.19.003240

Jae Woong Koh. Biomed J Sci \& Tech Res

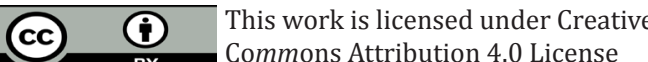

Submission Link: https://biomedres.us/submit-manuscript.php
10. Acharya NR, Srinivasan M, Mascarenhas J, Ravindran M, Zeqans M, et al. (2009) The steroid controversy in bacterial keratitis. Arch Ophthalmol 127(9): 1231.

11. Cohen EJ (2009) The case against the use of steroids in the treatment of bacterial keratitis. Arch Ophthalmol 127(1): 103-104.

12. Charukamnoetkanok P, Pineda R (2005) Controversies in management of bacterial keratitis. International Ophthalmology Clinics 45(4): 199210.

13. Aronson S, Moore T (1969) Corticosteroid therapy in central stromal keratitis. Ophthalmology 67(6): 873-896.

14. Carmichael T, Gelfand Y, Welsh N (1990) Topical steroids in the treatment of central and paracentral corneal ulcers. British Journal of Ophthalmology 74(9): 528-531.

15. Chusid MJ, Davis SD (1985) Polymorphonuclear leukocyte kinetics in experimentally induced keratitis. Arch Ophthalmol 103(2): 270-274.

16. Hindman HB, Patel SB, Jun AS (2009) Rationale for adjunctive topical corticosteroids in bacterial keratitis. Arch Ophthalmol 127(1): 97-102.

17. Wilhelmus KR, Gee L, Hauck WW, Kurinji N, Dawson CR, et al. (1994) Herpetic Eye Disease Study: a controlled trial of topical corticosteroids for herpes simplex stromal keratitis. Ophthalmology 101(12): 18831895.

18. Wilhelmus $\mathrm{K}$ (2002) Indecision about corticosteroids for bacterial keratitis: and evidence-based update. Ophthalmology 109(5): 835-842.

19. Stern G, Buttross M (1991) Use of corticosteroids in combination with antimicrobial drugs in the treatment of infectious corneal disease. Ophthalmology 98(6): 847-853.

20. Srinivasan M, Mascarenhas J, Rajaraman R, Ravindran M, Lalitha P, et al. (2012) Corticosteroids for bacterial keratitis: the Steroids for Corneal Ulcers Trial (SCUT). Arch Ophthalmol 130(2): 143-150.

21. Ray KJ, Srinivasan M, Mascarenhas J, Rajaraman R, Ravindran M, et al. (2014) Early addition of topical corticosteroids in the treatment of bacterial keratitis. JAMA Ophthalmol 132(6): 737-741.

22. McDonald TO, Borgmann AR, Roberts MD, Fox LG (1970) Corneal wound healing Inhibition of stromal healing by three dexamethasone derivatives. Investigative Ophthalmology \& Visual Science 9(9): 703709 .

23. Sugar J, Chandler JW (1974) Experimental corneal wound strength. Arch Ophthalmol 92(3): 248-249.

24. Srinivasan BD, Kulkarni PS (1981) The effect of steroidal and nonsteroidal anti-inflammatory agents on corneal re-epithelialization. Investigative Ophthalmology \& Visual Science 20(5): 688-691.

25. McGhee C, Dean S, Danesh-Meyer H (2002) Locally administered ocular corticosteroids: benefits and risks. Drug Safety 25(1): 33-55.

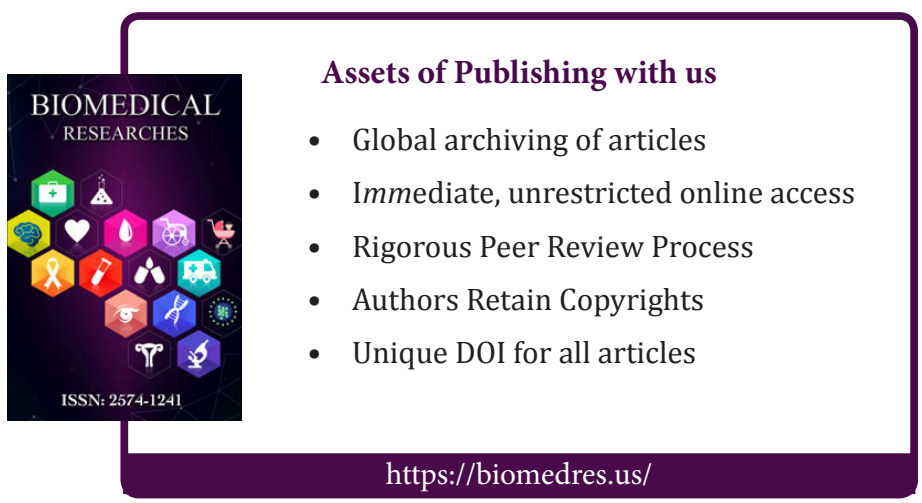

